\title{
COMPRESSION RATIO ESTIMATION BASED ON CYLINDER PRESSURE DATA
}

\author{
Marcus Klein, Lars Eriksson and Jan Åslund \\ Vehicular Systems, Dept of EE \\ Linköpings Universitet, SWEDEN \\ email:klein@isy.liu.se,larer@isy.liu.se,jaasl@isy.liu.se
}

\begin{abstract}
Four methods for compression ratio estimation of an engine from cylinder pressure traces are described and evaluated for both motored and fired cycles. The first three methods rely upon a model of polytropic compression for the cylinder pressure, and it is shown that they give a good estimate of the compression ratio for simulated cycles at low compression ratios. For high compression ratios, this simple model lack the information about heat transfer and the model error causes the estimates to become biased. Therefore a fourth method is introduced where heat transfer and crevice effects are modeled, together with a commonly used heat release model for firing cycles. This method is able to estimate the compression ratio more accurately at low as well as high compression ratios. Copyright (c)2004 IFAC
\end{abstract}

Keywords: Polytropic pressure model, nonlinear least squares, variable projection, compression ratio estimation, weighted least squares, parameter estimation, SI engine, variable compression

\section{INTRODUCTION}

A newly developed engine, which can continously change the compression ratio between 8 and 14 by tilting the mono-head, has been developed at SAAB Automobile AB. This ability to change the compression ratio opens up new opportunities to increase the efficiency of SI engines by down sizing and super charging. But if the compression ratio gets stuck at too high ratios, the risk of engine destruction by heavy knock increases rapidly. If the compression ratio gets stuck at too low ratios, we get an unnecessary low efficiency, and therefore an unnecessary high fuel consumption. It is therefore vital to monitor and diagnose the continuously changing compression ratio. Due to geometrical uncertainties, a spread of the compression ratio among the different cylinders is inherent [Amann, 1985], and since it is hard to measure the compression ratio directly, estimation is required. The questions asked here are related to: 1) accuracy, 2) convergence speed and 3) over all convergence. The approach investigated is to use cylinder pressure to estimate the compression ratio. A desirable prop- erty of the estimator is that it must be able to cope with the unknown offset introduced by the charge amplifier, changing thermodynamic conditions, and possibly also the unknown phasing of the pressure trace in relation to the crank angle revolution.

Two models of cylinder pressure with different complexity levels, a polytropic model and a singlezone zero-dimensional heat release model [Gatowski et al., 1984] are used. To estimate the parameters in the cylinder pressure models, three different optimization algorithms minimizing the prediction error are utilized, namely:

(1) A linear subproblem approach, where groups of the parameters are estimated one at a time and the predictor function is rewritten to be linear for the group of estimated parameters. Thus we can use linear regression at every substep for estimating the particular group of parameters.

(2) A variable projection method [Björck, 1996], where one iteration consists of two substeps: The first substep estimates the parameters that are linear in the predictor function, hold- 
ing the nonlinear constant. The second substep is to perform a line search in the direction of the negative gradient at the parameters found from substep one. This method classifies as a separable least squares method.

(3) Levenberg-Marquardt method, i.e. a GaussNewton method, where we here use numerical approximations of the gradient and the hessian.

Based on these models and optimization algorithms, four different methods are developed and used for compression ratio estimation for both motored and fired cycles.

\section{CYLINDER PRESSURE MODELING}

Two models are used for describing the cylinder pressure trace and they are refered to as the polytropic model and the standard model.

Polytropic model A simple but efficient model is the polytropic compression model,

$$
p(\theta) V(\theta)^{n}=C
$$

where $p$ is the cylinder pressure, $V(\theta)$ is the volume function, $n$ is the polytropic exponent and $C$ is a cycle-to-cycle dependent constant. The model is valid for adiabatic conditions, and works well during the compression and expansion phase of the engine cycle, but not during combustion [Heywood, 1988]. Therefore, for a firing cycle only data between inlet valve closing (IVC) and start of combustion (SOC) will be used, but for motored cycles all data during the closed part of the cycle, i.e. between IVC and exhaust valve opening (EVO), is utilized.

Standard model The article [Gatowski et al., 1984] develops, tests and applies the heat release analysis procedure used here. It maintains simplicity while still including the effects of heat transfer and crevice flows. The model has been widely used and the phenomena that it takes into account are well known [Heywood, 1988].

The pressure differential $d p$ can be written as

$$
d p=\frac{\delta Q_{c h}-\frac{\gamma}{\gamma-1} p d V-\delta Q_{h t}}{\frac{1}{\gamma-1} V+\frac{V_{c r}}{T_{w}}\left(\frac{T}{\gamma-1}-\frac{1}{b} \ln \left(\frac{\gamma-1}{\gamma^{\prime}-1}\right)+T^{\prime}\right)}
$$

This is an ordinary differential equation that easily can be solved numerically if a heat-release trace $\delta Q_{c h}$ is provided. The heat release is modeled by the Vibe function $x_{b}$ (3a) [Vibe, 1970]in its differentiated form $(3 \mathrm{~b})$.

$$
\begin{gathered}
x_{b}(\theta)=1-e^{-a\left(\frac{\theta-\theta_{0}}{\Delta \theta}\right)^{m+1}} \\
\frac{d}{d \theta} x_{b}(\theta)=\frac{a(m+1)}{\Delta \theta}\left(\frac{\theta-\theta_{0}}{\Delta \theta}\right)^{m} e^{-a\left(\frac{\theta-\theta_{0}}{\Delta \theta}\right)^{m+1}}
\end{gathered}
$$

Where $x_{b}$ is the mass fraction burned, $\theta_{0}$ is the start of the combustion, $\Delta \theta$ is the total combustion duration, and $a$ and $m$ are adjustable parameters. This model is valid between IVC and EVO.

\section{ESTIMATION METHODS}

\subsection{Cylinder pressure referencing}

Piezoelectric pressure transducers are used for measuring the in-cylinder pressure, which will cause a drift in the pressure trace. This drift is assumed to be constant during one engine cycle, and can be estimated with various methods [Randolph, 1990]. Here the measured pressure trace $p_{m}(\theta)$ will be referenced by comparing it to the intake manifold pressure $p_{\text {man }}$ just before inlet valve closing (IVC), for several samples of $p_{\text {man }}$. Due to standing waves in the intake runners at certain operating points, the referencing might prove to be insufficient, so a second pressure referencing is made for method 3 and 4 .

\subsection{Method 1 - Sublinear approach}

The first method estimates the polytropic exponent $n$, the compression ratio $r_{c}$ and the constant $C$ in the polytropic model. It iteratively solves two problems, one to determine the polytropic exponent $n$, and the other to determine the compression ratio $r_{c}$. By using the polytropic model (1)

$$
p(\theta)\left(V_{c}\left(r_{c}\right)+V_{d}(\theta)\right)^{n}=C
$$

the compression ratio $r_{c}$ can be estimated by iteratively estimating the clearance volume $V_{c}$ (i.e. $r_{c}$ ) and the polytropic exponent $n$.

Substep 1: The polytropic exponent $n$ is assumed to be known and the clearance volume is estimated by rewriting (4). This yields a least square problem which is linear in the parameters $C_{1}$ and $V_{c}$ according to

$$
V_{d}(\theta)=C_{1} p(\theta)^{-1 / n}-V_{c}\left(r_{c}\right)
$$

where $C_{1}=C^{1 / n}$ and $V_{c}$ are the parameters to be estimated.

Substep 2: The polytropic exponent $n$ is then estimated using the estimate of $V_{c}$ from substep 1 . Applying logarithms on (4) yields

$$
\ln p(\theta)=C_{2}-n \ln \left(V_{d}(\theta)+V_{c}\left(r_{c}\right)\right)
$$

which is linear in the parameters $n$ and $C_{2}=$ $\ln C$. The linear parameters are estimated and we return to substep 1 . This procedure is repeated until convergence.

Using this approach directly will cause diverging estimates [Klein and Eriksson, 2002]. This is due to that the predictor function is rewritten from (5) to (6) or vice versa in every substep. If the same predictor function could be used at every substep, the problem would be bilinear in the parameters and converge linearly [Björck, 1996].

In this case the predictor functions (5) and (6) are dissimilar in size. Taylor expansion shows that they become similar in size if (6) is multiplied by $\left(V_{c}\left(r_{c}\right)+V_{d}(\theta)\right)$

$\left(V_{c}+V_{d}(\theta)\right) \ln p(\theta)=\left(V_{c}+V_{d}(\theta)\right)\left(C_{2}-n \ln \left(V_{d}(\theta)+V_{c}\right)\right)$ 
Stoping criteria: This modification (7) stabilizes the method in the sense that the parameter estimates after a few iterations jumps back and forth between two parameter vectors. The jumping is due to that the norm of the two predictor functions are not exactly the same given the same parameters. However, at this stage the components of the two vectors differ less than $0.1 \%$ and can therefore be considered to be the same. By doing so and setting the stoping criteria to a parameter change less than $0.1 \%$, the method stops within two iterations. This limit is not universally valid, and must be adapted for every specific engine and measurement setup. Also note that convergence can not be guaranteed for this method. If the clearance volume $V_{c}$ is known, the same method can be used to instead estimate an additive pressure bias by reforming the weighting function in a straight-forward manner.

\subsection{Method 2 - Variable projection}

The second method also uses the polytropic compression model (1), together with a variable projection algorithm. A nonlinear least squares problem $\min _{x}\|r(x)\|^{2}$ is separable if the parameter vector $x$ can be partitioned such that $x=(y z)^{T}$

$$
\min _{y}\|r(y, z)\|_{2}
$$

is easy to solve. If $r(y, z)$ is linear in $y, r(y, z)$ can be rewritten as

$$
r(y, z)=F(z) y-g(z)
$$

For a given $z$, this is minimized by

$$
y(z)=\left[F^{T}(z) F(z)\right]^{-1} F(z) g(z)=F^{\dagger}(z) g(z)
$$

using linear regression. The original problem $\min _{x}\|r(x)\|^{2}$ can then be rewritten as

$$
\min _{z}\|r(y, z)\|_{2}=\min _{z}\|g(z)-F(z) y(z)\|_{2}
$$

and

$$
\begin{aligned}
r(y, z) & =g(z)-F(z) y(z)=g(z)-F(z) F^{\dagger}(z) g(z) \\
& =\left(I-P_{F(z)}\right) g(z)
\end{aligned}
$$

where $P_{F(z)}$ is the orthogonal projection onto the range of $F(z)$, thus the name variable projection method.

The polytropic model in (4) is rewritten as

$$
\ln p(\theta)=C_{2}-n \ln \left(V_{d}\left(\theta^{\prime}\right)+V_{c}\right)
$$

which is the same equation as (6). This equation is linear in the parameters $C_{2}=\ln C$ and $n$ and nonlinear in $V_{c}$ and applies to the form given in (A.1). A computationally efficient algorithm [Björck, 1996, p.352] is summarized for our application in Appendix A. For this application the method converges within four iterations.

\subsection{Method 3 - Levenberg-Marquardt}

The third method uses the polytropic compression equation (1) as methods 1 and 2 did, but a pressure sensor model is added according to

$$
p(\theta)=p_{m}(\theta)+\Delta p
$$

in order to make the pressure referencing better. The crank angle phasing $\Delta \theta$ of the volume and pressure traces is also included in the polytropic model, which then can be written as

$p(\theta)=p_{m}\left(\theta^{\prime}+\Delta \theta\right)+\Delta p=C \cdot\left(V_{d}\left(\theta^{\prime}+\Delta \theta\right)+V_{c}\right)^{-n}$

Based on (15) the following nonlinear least squares problem is formulated

$\min _{x} \sum_{i=1}^{N}\left(p_{m}\left(\theta_{i}\right)+\Delta p-C \cdot\left(V_{d}\left(\theta_{i}+\Delta \theta\right)+V_{c}\right)^{-n}\right)^{2}$

A Levenberg-Marquardt method [Gill et al., 1981] is used to solve this nonlinear least squares problem. The problem has good numerical properties, the Levenberg-Marquardt method has second order local convergence and for this application the method converges within ten iterations.

\subsection{Method 4 - Levenberg-Marquardt and standard model}

The fourth method uses the single zone model (2) from [Gatowski et al., 1984] which includes heat transfer and crevice effects, and it will be used to improve the estimation accuracy. The free parameters are summarized in [Klein and Eriksson, 2002]. Due to the complexity of this model, the sublinear approach and variable projection approach are not suitable for optimization, and therefore only the Levenberg-Marquardt method is used. This approach has earlier been successfully applied in [Eriksson, 1998] for motoring cycles, but here a heat-release model (3b) is included to cope with firing cycles.

\subsection{Summary of methods}

The following table shows the relation between the different methods.

\begin{tabular}{l|c|c} 
& Polytropic Model & Standard Model \\
\hline Opt alg 1 & Method 1 & \\
\hline Opt alg 2 & Method 2 & \\
\hline Opt alg 3 & Method 3 & Method 4 \\
\hline
\end{tabular}

For firing cycles, methods 1,2 and 3 will only use cylinder pressure data between IVC and SOC, in contrast to method 4 which will use data from the entire closed part of the engine cycle. For motoring cycles, all data during the closed part of the cycle is utilized by all methods. 


\section{SIMULATION RESULTS}

Since the true values of the compression ratios of the engine are unknown, simulations of the cylinder pressure trace are necessary to perform and use for evaluating the four proposed methods. Only then can it be determined whether the estimates are accurate (unbiased) or not.

Cylinder pressure simulations were made using the standard model (2) with representative parameters [Klein and Eriksson, 2002]. Sixty realizations of Gaussian noise with zero mean and standard deviation $2 \mathrm{kPa}$ were added to the simulated cylinder pressures. The following sections show the typical behavior of the estimation methods for a representative cycle at a high compression ratio $r_{c}=13$ displayed in Figure 1, where the effects of heat transfer are more likely to show due to the higher pressure and temperature in the cylinder. Residuals

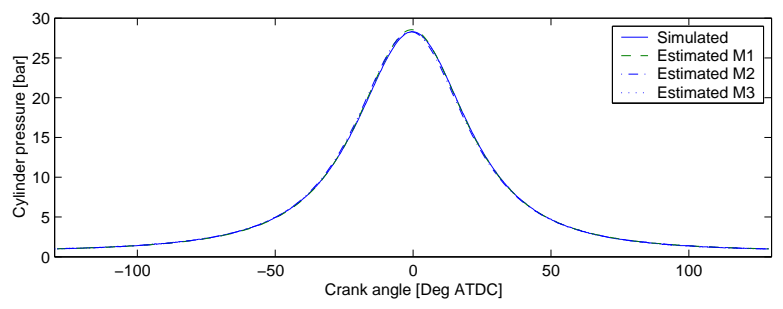

Fig. 1. Simulated cylinder pressure for a motored cycle at $r_{c}=13$.

for all methods are found in figures 2 to 4 and figures 5 and 6 show a summary of all estimations for motored and fired cycles respectively. In the summary section, statistics of the performance for the four methods are summarized for both firing and motoring cycles.

\subsection{Method 1}

Method 1 converges within two iterations for almost all simulated cylinder pressures, both firing and motored cycles. Using the simulated cylinder pressure an estimation of the parameters is made, and from these a residual from the simulated and estimated cylinder pressure can be formed. In Figure 2, the residual corresponding to the cylinder pressure in Figure 1 for method 1 is shown. At the beginning of the compression phase and at the end of the expansion, the model and estimation method works satisfactory, but not in between where most of the heat transfer occurs. This model inaccuracy is partly covered by allowing the polytropic exponent to be small.

\subsection{Methods 2 and 3}

Methods 2 and 3 show the same lack of model accuracy as method 1, although the residuals for $r_{c}=13$ (Figure 2) do not look the same and the estimated $r_{c}$ is biased. But when lowering the compression ratio the model becomes more accurate,
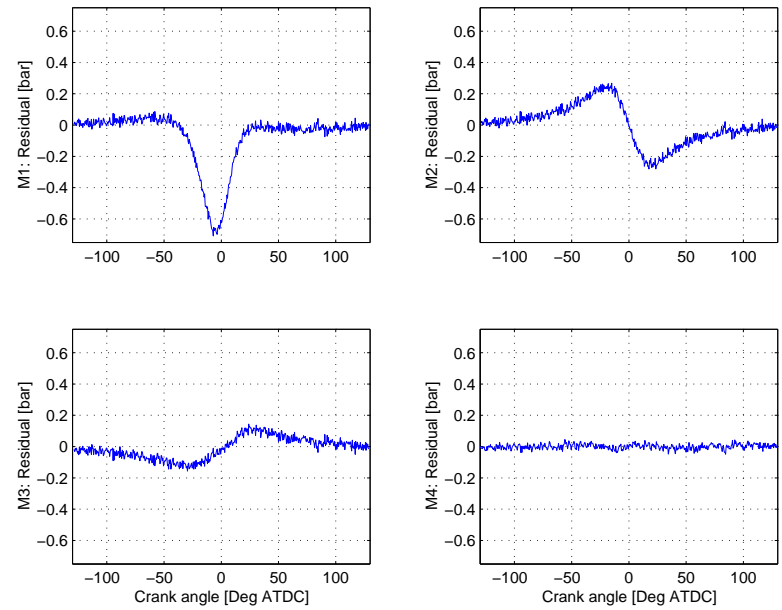

Fig. 2. Difference between estimated and simulated cylinder pressure for all methods, given the motored cycle in Figure 1.

and the residual in Figure 3 does not show the same systematic deviation as the corresponding residual in Figure 2 did. This is due to that the heat transfer
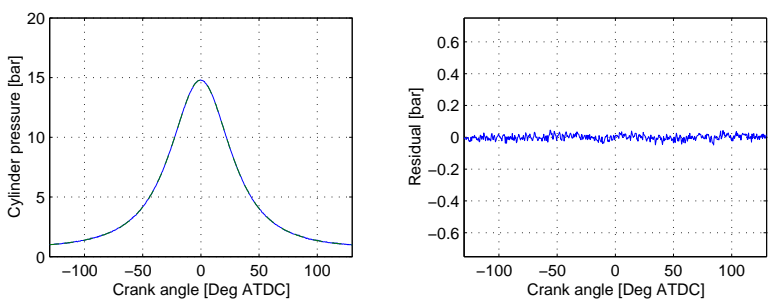

Fig. 3. Difference between estimated and simulated cylinder pressure using method 3, for a motored cycle at $r_{c}=8$.

and crevice effects are smaller, due to the lower pressure and temperature rendering from the lower compression ratio. The systematic deviation for the residuals are expected since the crevice flow and heat transfer are not considered explicitly in the polytropic model (1) and it stresses that these phenomena must be taken into account when a better estimate is desired for higher compression ratios. Therefore method 4 uses the standard model (2).

\subsection{Method 4}

Reasonable enough, the more complex method also shows the best ability to adjust to the simulated cylinder pressure and explain the physical phenomena taking place in the cylinder. The residual for method 4 in Figure 2 looks like white noise, suggesting that the estimation method can explain the data fully. This is also the case for firing cycles, see Figure 4 where the simulated and estimated cylinder pressure is shown together with the residual.

\subsection{Summary of parameter estimations}

Comparing the residuals from all methods, it is obvious that method 4 can explain the data most 

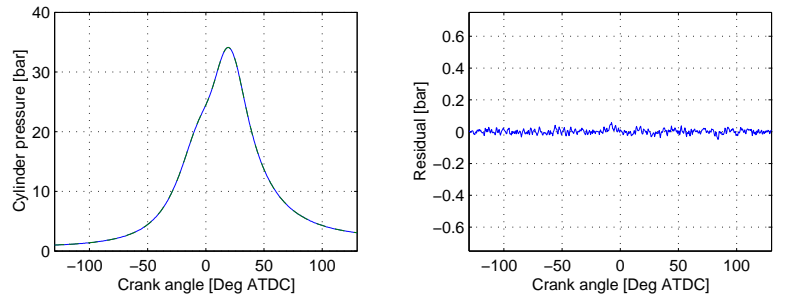

Fig. 4. Difference between estimated and simulated cylinder pressure using method 4 , for a fired cycle at $r_{c}=13$.

accurately. This suggests that the estimation of the compression ratio becomes best for method 4, which is also shown in Figure 5 for motored cycles, where the mean and $95 \%$ confidence interval of the estimated compression ratio is shown for all four methods. The $95 \%$ confidence interval is computed by assuming that the model is correct and that the estimation error asymptotically converges to a Gaussian distribution. In the figure the real com-

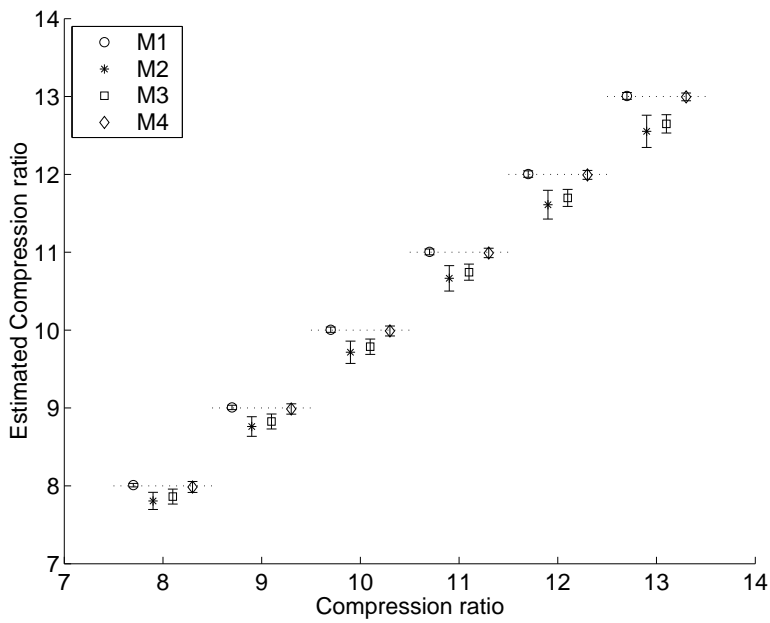

Fig. 5. Mean and 95\% confidence interval of the estimated compression ratio for motored cycles using the four methods, compared to the real compression ratio.

pression ratios are the integer values 8 til 13 and for convenience, method 1 is moved to the left, method 2 is moved a little to the left (and to the right of method 1), method 3 is moved a little to the right and method 4 is to the right of method 3 . The estimates should be as close to the horizontal lines as possible.

Method 1 estimates the compression ratio very well, almost equally well as method 4. As mentioned before, this is allowed by letting the polytropic coefficient become small. Methods 2 and 3 on the other hand under estimates the compression ratio, and this systematic fault increases with the compression ratio. Method 4 is able to estimate the compression ratio correctly, due to the higher flexibility of the model. This suggests that method 1 or 4 should be used.

For firing cycles the same effect as for the motoring cycles appears and is even more pronounced as shown in Figure 6. Table 1 summarizes the standard

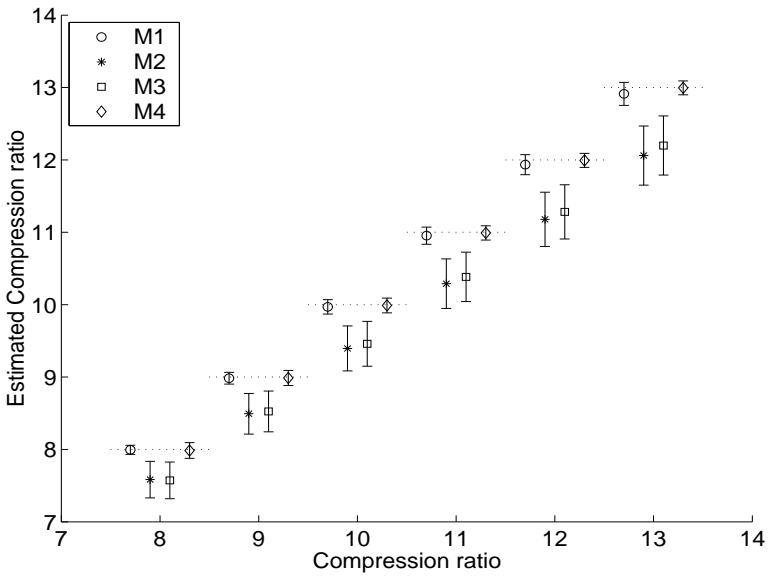

Fig. 6. Mean and 95\% confidence interval of the estimated compression ratio for fired cycles using the four methods, compared to the real compression ratio.

deviation and the maximum relative error of the estimated compression ratio for 60 cycles respectively. The mean and maximum error is smallest for method 4 both for firing and motoring cycles. The standard deviation is small for all methods.

\begin{tabular}{cl|l|l|l}
\multirow{2}{*}{ Method } & Type & Std dev & \multicolumn{2}{|c}{ Rel error (\%) } \\
& & & Max & Mean \\
\hline \multirow{2}{*}{1} & Fired & 0.0443 & 1.34 & 0.37 \\
& Motored & 0.0226 & 0.47 & 0.064 \\
\hline \multirow{2}{*}{2} & Fired & 0.1236 & 3.96 & 6.2 \\
& Motored & 0.0243 & 2.02 & 2.9 \\
\hline \multirow{2}{*}{3} & Fired & 0.1284 & 2.51 & 5.6 \\
& Motored & 0.0112 & 1.44 & 2.2 \\
\hline \multirow{2}{*}{4} & Fired & 0.0318 & 0.49 & 0.17 \\
& Motored & 0.0156 & 0.29 & 0.099 \\
\hline
\end{tabular}

Table 1. Table showing the standard deviation, maximum and mean relative error of the estimated compression ratio $r_{c}$.

\subsection{Small study of varying operating conditions}

To investigate how the proposed methods behave for various operating conditions, a small study is performed using the cylinder pressures in Figure 7. Table 2 summarizes the results. Method 4 is not included, since it is able to estimate $r_{c}$ correctly. Method 1 performs better than methods 2 and 3, although the estimate becomes worse for higher load engine operating conditions. But it is still within $2 \%$ for a high engine load level (OP3).

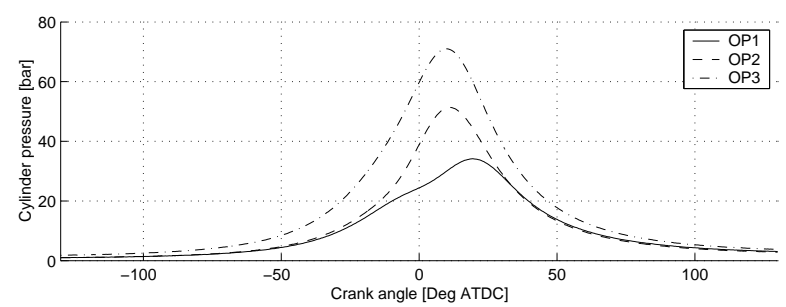

Fig. 7. Simulated cylinder pressure for firing cycles at $r_{c}=13$ for three operating points. 


\begin{tabular}{|c|l|l|l|l|l|l|}
\hline Oper & \multicolumn{2}{|c|}{ M1 } & \multicolumn{2}{c|}{ M2 } & \multicolumn{2}{c|}{ M3 } \\
point & $@ 8$ & $@ 13$ & @8 & @13 & $@ 8$ & $@ 13$ \\
\hline OP1 & 0.1 & 0.7 & 5.2 & 7.2 & 5.3 & 6.2 \\
OP2 & 0.5 & 1.7 & 9.1 & 12.4 & 9.3 & 10.6 \\
OP3 & 0.8 & 2.0 & 8.1 & 11.3 & 8.3 & 9.6 \\
\hline
\end{tabular}

Table 2. Relative estimation mean error

[\%] in $r_{c}$ using methods 1, 2 and 3 for firing cycles.

\subsection{Conclusions from simulations}

The first three methods rely upon the assumption of a polytropic compression and expansion, and it is shown that this is sufficient to get a rough estimate of the compression ratio, especially for low compression ratios and by letting the polytropic exponent to become small. But for higher $r_{c}$ :s it is important to take the heat transfer into account, and then only method 4 is accurate enough. It is interesting to note that for diagnostic purposes, all four methods will be able to detect when the compression ratio gets stuck at a too high or too low level.

The time complexity for the four methods is quite diverse, and is summarized in table 3 . The simulations were made using Matlab 6.1 on a SunBlade 100, which has a 64-bit $500 \mathrm{Mhz}$ processor.

\begin{tabular}{c|l|c|c} 
Method & Time & \# Iter & \# Parameters \\
\hline 1 & $15.4 \mathrm{~ms}$ & 2 & 3 \\
\hline 2 & $23 \mathrm{~ms}$ & 3 & 3 \\
\hline 3 & $145 \mathrm{~ms}$ & 5 & 5 \\
\hline 4 & $2 * 10^{5} \mathrm{~ms}$ & 9 & 12 \\
\hline Table 3. Table showing the mean time
\end{tabular}

Table 3. Table showing the mean time and mean number of iterations in completing one cycle, together with the num-

ber of parameters for all methods.

\section{CONCLUSIONS}

The four estimation methods all give good estimations for low compression ratios on simulated data. But for high compression ratios, the heat transfer has to be accounted for and therefore the more complex method 4 gives a better estimation than the simpler and more time efficient methods 1,2 and 3. Method 2 is preferable to method 3 due to its faster convergence. During driving, all methods are able to detect if the compression ratio is stuck at a too high or at a too low level. This is sufficient both for safety reasons, where the compression ratio can be too high and engine knock is the consequence, and for fuel economic reasons, where a too low compression ratio will lead to higher fuel consumption. However, to fully validate the proposed methods, an experimental evaluation with real engine data is required.

\section{References}

C.A. Amann. Cylinder-pressure measurement and its use in engine research. SAE Technical Paper 852067, 1985.
Å. Björck. Numerical Methods for Least Squares Problems. Siam, 1996.

L. Eriksson. Requirements for and a systematic method for identifying heat-release model parameters. (SAE Technical Paper no.980626), 1998.

J. A. Gatowski, E. N. Balles, K. M. Chun, F. E. Nelson, J. A. Ekchian, and J. B. Heywood. Heat release analysis of engine pressure data. $S A E$ Technical Paper 841359, 1984.

P.E. Gill, W. Murray, and M.H. Wright. Practical Optimization. Academic Press, 1981.

J. B. Heywood. Internal Combustion Engine Fundamentals. McGraw-Hill series in mechanical engineering. McGraw-Hill, 1988. ISBN 0-07-1004998.

M. Klein and L. Eriksson. Models, methods and performance when estimating the compression ratio based on the cylinder pressure. CCSSE, October 2002.

A.L. Randolph. Methods of processing cylinderpressure transducer signals to maximize data accuracy. SAE Technical Paper 900170, 1990.

I.I. Vibe. Brennverlauf und Kreisprocess von Verbennungsmotoren. VEB Verlag Technik Berlin, 1970. German translation of the russian original.

\section{Appendix A. VARIABLE PROJECTION ALGORITHM}

A computationally efficient algorithm is described in [Björck, 1996] and is summarized here. Partition the parameter vector $x$ such that $x=(y z)^{T}$, where $r(y, z)$ is linear in $y$. Rewrite $r(y, z)$ as

$$
r(y, z)=F(z) y-g(z)
$$

Let $x_{k}=\left(y_{k}, z_{k}\right)$ be the current approximation.

(1) Solve the linear subproblem

$$
\min _{\delta y_{k}}\left\|F\left(z_{k}\right) \delta y_{k}-\left(g\left(z_{k}\right)-F\left(z_{k}\right) y_{k}\right)\right\|_{2}
$$

and set $x_{k+1 / 2}=\left(y_{k}+\delta y_{k}, z_{k}\right)$.

(2) Compute the Gauss-Newton direction $p_{k}$ at $x_{k+1 / 2}$, i.e. solve

$$
\min _{p_{k}} \| C\left(x_{k+1 / 2} p_{k}+r\left(y_{k+1 / 2}, z_{k}\right) \|_{2}\right.
$$

where $C\left(x_{k+1 / 2}=\left(F\left(z_{k}\right), r_{z}\left(y_{k+1 / 2}, z_{k}\right)\right)\right.$ is the Jacobian matrix.

(3) Set $x_{k+1}=x_{k+1 / 2}+\alpha_{k} p_{k}$ and return to step 1.

The polytropic model in (4) is rewritten as

$$
\ln p(\theta)=C_{2}-n \ln \left(V_{d}\left(\theta^{\prime}\right)+V_{c}\right)
$$

This equation is linear in the parameters $C_{2}=\ln C$ and $n$ and nonlinear in $V_{c}$ and applies to the form given in (A.1). With the notation from the algorithm above, the parameters are $x=\left(C_{2} n V_{c}\right)^{T}$, where $y=\left(C_{2} n\right)^{T}$ and $z=V_{c}$. The measurement vector is formed as $g=-\ln p$ and the regression vector as $F=\left[-I \ln \left(V_{c}+V_{d}\left(\theta^{\prime}\right)\right)\right]$. 